\section{Aspectos metodológicos do Projeto SBBrasil 2010 de interesse para inquéritos nacionais de saúde}

\author{
Relevant methodological issues from the SBBrasil \\ 2010 Project for national health surveys
}

\author{
Angelo Giuseppe Roncalli 1 \\ Nilza Nunes da Silva 2 \\ Antonio Carlos Nascimento ${ }^{3}$ \\ Cláudia Helena Soares de Morais Freitas 4 \\ Elisete Casotti 5 \\ Karen Glazer Peres 6 \\ Lenildo de Moura 7,8 \\ Marco A. Peres 6 \\ Maria do Carmo Matias Freire 9 \\ Maria Ilma de Souza Cortes 10 \\ Mario Vianna Vettore 11 \\ Moacir Paludetto Júnior 12 \\ Nilcema Figueiredo 13 \\ Paulo Sávio Angeiras de Goes 14 \\ Rafaela da Silveira Pinto 15 \\ Regina Auxiliadora de Amorim Marques 16,17 \\ Samuel Jorge Moysés 18,19 \\ Sandra Cristina Guimarães Bahia Reis 20,21 \\ Paulo Capel Narvai ${ }^{2}$
}

\title{
Introdução
}

${ }_{1}$ Centro de Ciências da Saúde, Universidade Federal do Rio Grande do Norte, Natal, Brasil.

2 Faculdade de Saúde Pública, Universidade de São Paulo, São Paulo, Brasil. 3 Faculdade de Odontologia, Universidade Positivo, Curitiba, Brasil.

${ }_{4}^{4}$ Centro de Ciências da Saúde, Universidade Federal da Paraíba, João Pessoa, Brasil.

5 Universidade Severino Sombra, Vassouras, Brasil. ${ }_{6}$ Centro de Ciências da Saúde, Universidade Federal de Santa Catarina, Florianópolis, Brasil.

7 Secretaria de Vigilância em Saúde, Ministério da Saúde, Brasília, Brasil.

8 Universidade Federal do Rio Grande do Sul, Porto Alegre, Brasil.

Outras instituições listadas ao final do artigo.

\section{Correspondência}

A. G. Roncalli

Departamento de Odontologia, Centro de Ciências da Saúde, Universidade Federal do Rio Grande do Norte. Av. Salgado Filho 1787, Natal, RN 59056-000, Brasil. roncalli@terra.com.br

\section{Abstract}

The SBBrasil 2010 Project (SBB10) was designed as a nationwide oral health epidemiological survey within a health surveillance strategy. This article discusses methodological aspects of the SBB10 Project that can potentially help expand and develop knowledge in the health field. This was a nationwide survey with stratified multistage cluster sampling. The sample domains were 27 State capitals and 150 rural municipalities (counties) from the country's five major geographic regions. The sampling units were census tracts and households for the State capitals and municipalities, census tracts, and households for the rural areas. Thirty census tracts were selected in the State capitals and 30 municipalities in the countryside. The precision considered the demographic domains grouped by density of the overall population and the internal variability of oral health indices. The study evaluated dental caries, periodontal disease, malocclusion, fluorosis, tooth loss, and dental trauma in five age groups (5, 12, 15-19, 35-44, and 65-74 years).

Oral Health; Epidemiologic Surveillance; CrossSectional Studies
Levantamentos epidemiológicos de saúde bucal vêm sendo realizados, periodicamente, no Brasil desde o início dos anos 1950, no âmbito dos denominados "programas incrementais" 1. Embora com abrangência restrita à população escolar coberta por tais programas, essas iniciativas possibilitaram desenvolver e consolidar no país, importantes conhecimentos epidemiológicos. Contudo, inquéritos de saúde bucal de abrangência nacional se tornaram realidade apenas a partir da segunda metade dos anos 1980, com a realização do primeiro levantamento epidemiológico em saúde bucal em 1986. Dez anos depois, foi realizado o segundo inquérito nacional, em 1996 2,3. A despeito da grande importância que tiveram a seu tempo, esses estudos não se estabeleceram enquanto um componente da política de saúde bucal vigente 3 .

A experiência seguinte se deu com o Projeto SB 2000 (SB2000), desenvolvido para gerar informações que permitissem avaliar a situação do país em relação às Metas em Saúde Bucal para o Ano 2000, propostas pela Organização Mundial da Saúde (OMS) 4. O SB2000 teve como base a metodologia proposta pela OMS no final dos anos 19905 com relação às faixas etárias e índices utilizados, porém trabalhou com um desenho amostral específico, tendo em conta a complexidade territorial brasileira. O levantamento só 
foi concluído em 2003, razão pela qual o estudo, inicialmente SB2000, ficou conhecido como SBBrasil 2003 (SBB03). Não obstante essas dificuldades operacionais, o SBB03 representou um expressivo avanço nos inquéritos nacionais de saúde bucal, consolidando-os no âmbito do Sistema Único de Saúde (SUS) 6.

Em 2009, no escopo da construção do eixo da vigilância em saúde da Política Nacional de Saúde Bucal, foi proposta a edição 2010 do SBBrasil (SBB10), a qual aperfeiçoou a proposta metodológica inaugurada pelo SBB03, constituindo-se na principal estratégia de vigilância em saúde bucal, com base na produção de dados primários, contribuindo desse modo para a construção de uma Política Nacional de Saúde Bucal pautada em modelos de atenção de base epidemiológica. Cabe assinalar que o SBB03 incorporou elementos da metodologia proposta pela OMS para inquéritos de saúde bucal, mas não se ateve apenas às recomendações do manual, indo além e compondo um desenho de estudo mais adequado à realidade nacional e com características complexas. Já o SBB10 manteve boa parte da estratégia metodológica do SBB03, porém avançou em alguns pontos considerados insuficientes na experiência anterior 6. Desse modo, o objetivo deste artigo é apresentar os principais aspectos metodológicos do SBB10, com a finalidade de registrar o desenvolvimento histórico acumulado nessa área, colaborar na superação de dificuldades que venham a ser encontradas em iniciativas congêneres e contribuir para o aperfeiçoamento e consolidação do próprio Projeto SBBrasil.

\section{Características da pesquisa}

Os estudos seccionais de abrangência nacional no Brasil se constituem em grandes desafios, dadas as características peculiares do país, com uma vasta extensão territorial e uma grande heterogeneidade entre as regiões. Desde 1969, o Instituto Brasileiro de Geografia e Estatística (IBGE) estabeleceu a divisão territorial brasileira em 5 blocos formados com base em critérios naturais como clima, relevo, vegetação e hidrografia, sendo, por esta razão, chamadas de "regiões naturais": Norte, Nordeste, Sudeste, Sul e Centrooeste. Embora tenha sido formada por critérios eminentemente geográficos, a divisão regional brasileira, pelo modo como se deu o processo de colonização e desenvolvimento do país, também expressa diferentes características econômicas e culturais. Desse modo, é relativamente comum se utilizar essa divisão como primeira estratificação em estudos nacionais, e foi essa a opção do SBB10.
Por outro lado, e também pelas mesmas razões ligadas ao desenvolvimento, as capitais dos 26 estados, juntamente com o Distrito Federal, aglutinam quase um quarto de toda a população do país (mais de 45 milhões de pessoas). É também o espaço onde se concentra a maior parte da atividade econômica, de modo que, independentemente da região em que esteja localizada, uma capital se constitui em um locus bastante peculiar e que deve ser considerado em qualquer estudo de base nacional.

Nesse sentido, do ponto de vista de organização geral, o SBB10 se constitui em uma pesquisa de base nacional, com representatividade para as capitais de estado e o Distrito Federal, e para as cinco regiões naturais (Norte, Nordeste, Sudeste, Sul e Centro-oeste). Compõe um estudo com base em uma amostra de indivíduos residentes em 177 municípios, nos quais foram realizados exames bucais para avaliar a prevalência e a gravidade dos principais agravos bucais, e aplicados questionários para coleta de dados sobre a condição socioeconômica, utilização de serviços odontológicos e percepção de saúde.

Com relação ao componente operacional, a pesquisa se constitui em um estudo coordenado e financiado pelo Ministério da Saúde, contando com a participação articulada das Secretarias de Atenção à Saúde (SAS) e de Vigilância à Saúde (SVS). Na execução, teve a participação ativa das Secretarias Estaduais e Municipais de Saúde e contou com o apoio de entidades odontológicas, universidades e institutos de pesquisa, articulados pela Coordenação Nacional de Saúde Bucal, por intermédio do seu Comitê Técnico Assessor para Vigilância em Saúde Bucal (CTA) e dos Centros Colaboradores em Vigilância em Saúde Bucal (Cecol). O Projeto teve, ainda, o apoio da Associação Brasileira de Pós-graduação em Saúde Coletiva (ABRASCO) e a colaboração do IBGE.

\section{Plano amostral}

\section{Domínios do estudo}

O Plano Amostral constou de domínios relativos às capitais e municípios do interior. Cada capital de Unidade da Federação (estados e Distrito Federal) compôs um domínio e cada região um outro, representativo dos municípios do interior. Desse modo, tem-se, ao todo, 27 domínios de capital, mais 5 domínios de interior, um para cada região, totalizando 32 (Figura 1). 
Figura 1

Esquema do processo de composição da amostra.

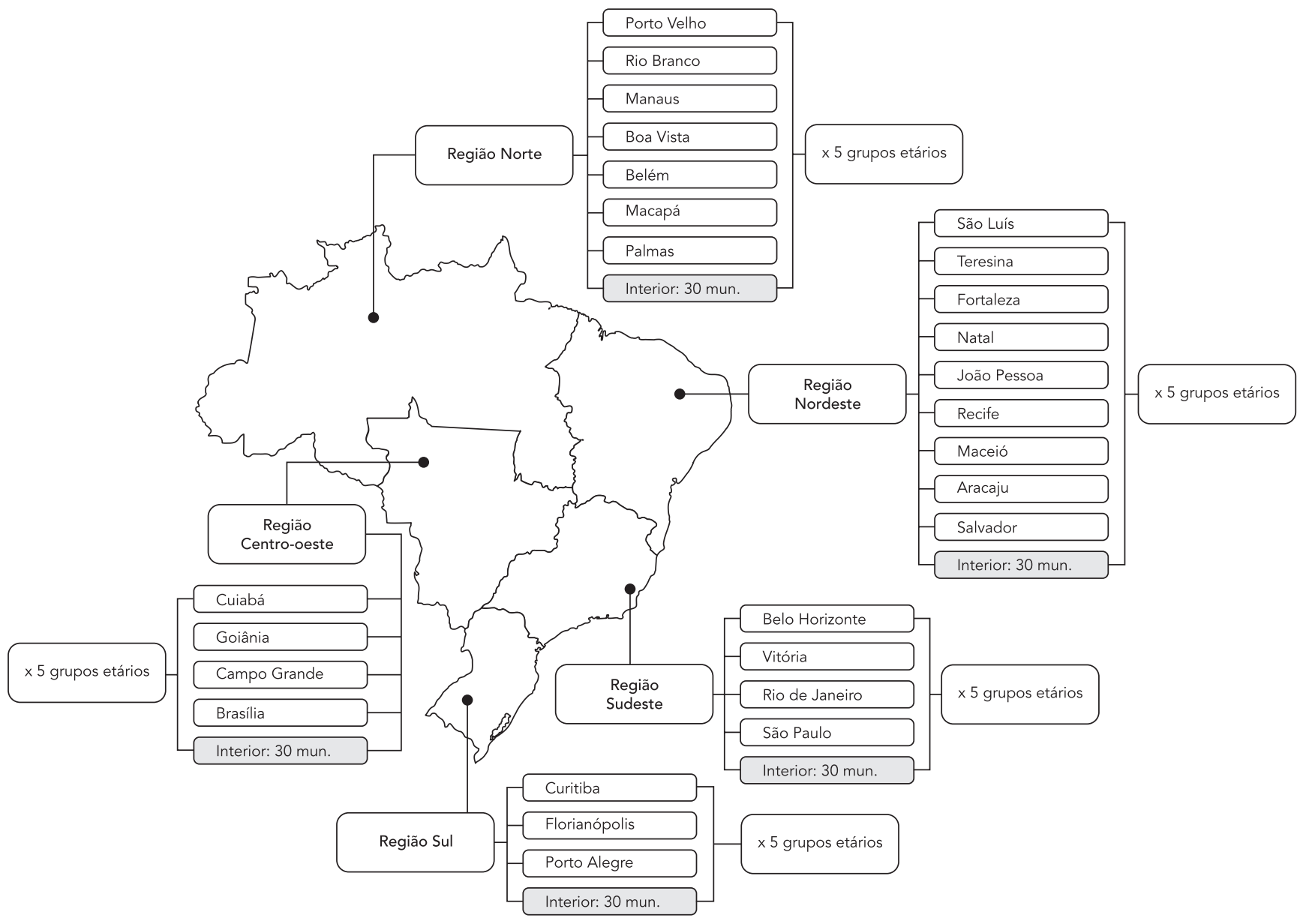

\section{Idades e grupos etários-índice}

A OMS sugere, para estudos de saúde bucal, a composição da amostra em determinadas idades e grupos etários-índice, tendo em vista serem suficientemente capazes de expressar as condições das demais idades e grupos etários. No SBB10 essa recomendação foi aceita, com algumas modificações. As descrições apresentadas a seguir foram retiradas parcialmente da 4a edição do Manual da OMS, de 19975.

\section{- 5 anos}

Esta idade é de interesse em relação aos níveis de doenças bucais na infância, uma vez que pode exibir mudanças em um período de tempo menor que a dentição permanente em outras idades-índice. É usada internacionalmente para aferição do ataque de cárie em dentes decíduos.

\section{- 12 anos}

Esta idade é especialmente importante, tendo sido escolhida como a idade de monitoramento global da cárie, para comparações internacionais e o acompanhamento das tendências da doença.

\section{- 15 a 19 anos}

Considerando a possibilidade de comparação com os dados de 1986 e levando-se em conta, ainda, que ao se trabalhar com idades restritas como 15 ou 18 anos aumentam expressivamente 
as dificuldades para compor a amostra (em função das proporções dessas idades - 15 ou 18 anos - no conjunto da população), decidiu-se manter a faixa de 15 a 19 anos.

\section{- 35 a 44 anos}

Este grupo é o padrão para avaliar condições de saúde bucal em adultos, como o efeito total da cárie dentária, o nível de gravidade do envolvimento periodontal ou os efeitos gerais dos tratamentos prestados.

\section{- 65 a 74 anos}

Este grupo etário tem se tornado mais importante com as mudanças na distribuição etária e no aumento da expectativa de vida que vem ocorrendo também no Brasil. Dados desse grupo são cada vez mais importantes tanto para o planejamento em saúde como para o monitoramento dos efeitos gerais dos serviços odontológicos prestados à população.

\section{Unidades Primárias de Amostragem}

A escolha de uma eficiente Unidade Primária de Amostragem (UPA) em estudos seccionais é um aspecto importante no estabelecimento da sua qualidade, uma vez que afeta todas as fases do estudo. Nesse sentido, devem, de uma maneira geral, ter limites claros e estáveis durante certo período, cobrir totalmente a população alvo do estudo, ter tamanho adequado, possuir dados disponíveis para eventuais processos de estratificação e ser em número suficiente, dentro do domínio do estudo 7 .

No caso do Brasil, em pesquisas de base domiciliar, os setores censitários preenchem de modo bastante satisfatório esses requisitos. $\mathrm{O}$ setor censitário é definido como a unidade de controle cadastral formada por área contínua, situada em um único quadro urbano ou rural, com dimensão e número de domicílios ou de estabelecimentos definidos, sendo a média nacional de aproximadamente 300 domicílios ${ }^{8}$. Operacionalmente, o setor censitário é a unidade territorial sobre a qual foram realizados os percursos para sorteio e identificação dos domicílios.

O processo de amostragem por conglomerados 9,10 foi, então, estruturado em dois estágios, para os municípios de capitais, e em três, para o interior das cinco regiões brasileiras. As unidades amostrais foram, respectivamente, setor censitário e domicílio para as capitais, e município, setor censitário e domicílio para o interior.

Em cada domínio geográfico foram utilizadas 30 UPA. Nas capitais foram sorteados 30 setores censitários, enquanto que no interior de cada região foram 30 municípios (Tabela 1).

\section{- Sorteios das UPA - Interior}

Os 30 municípios em cada região foram sorteados pela técnica PPT (Probabilidade Proporcional ao Tamanho) 9,10. Uma lista com todos os municípios e respectiva população estimada para 2009 foi obtida junto ao IBGE e exportada para uma planilha Excel (Microsoft Corp.). Foram excluídas as capitais e criadas planilhas separadas para cada região. Em cada planilha, os municípios foram ordenados inicialmente por estado (código do IBGE) e, em seguida, pelo tamanho de sua população. A população total dos municípios do interior de uma dada região foi então calculada e este total foi dividido por 32 (30 municípios titulares, mais dois municípios de reserva), obtendose, desta forma, o intervalo de amostragem. Os municípios sorteados duplamente ("autosorteados”) foram excluídos e novo intervalo foi calculado. Esse processo foi repetido em cada uma das cinco regiões, de modo a compor a amostra final de 150 municípios do interior.

Somando-se os municípios do interior às 27 capitais de unidades da federação, o número total de municípios da amostra ficou em 177. A Figura 2 mostra a distribuição dessa amostra, distinguidos por porte populacional.

Tabela 1

Estágios e domínios no processo de amostragem.

\begin{tabular}{|c|c|c|}
\hline \multirow[t]{2}{*}{ Estágios } & \multicolumn{2}{|c|}{ Domínio } \\
\hline & Capital & Interior \\
\hline 1ㅇ Estágio (UPA) & Setor Censitário (30 em cada capital) & Município (30 em cada região) \\
\hline 2o Estágio & Domicílio & Setor Censitário (2 em cada município sorteado) \\
\hline 3으 Estágio & & Domicílio \\
\hline
\end{tabular}

UPA: Unidade Primária de Amostragem. 


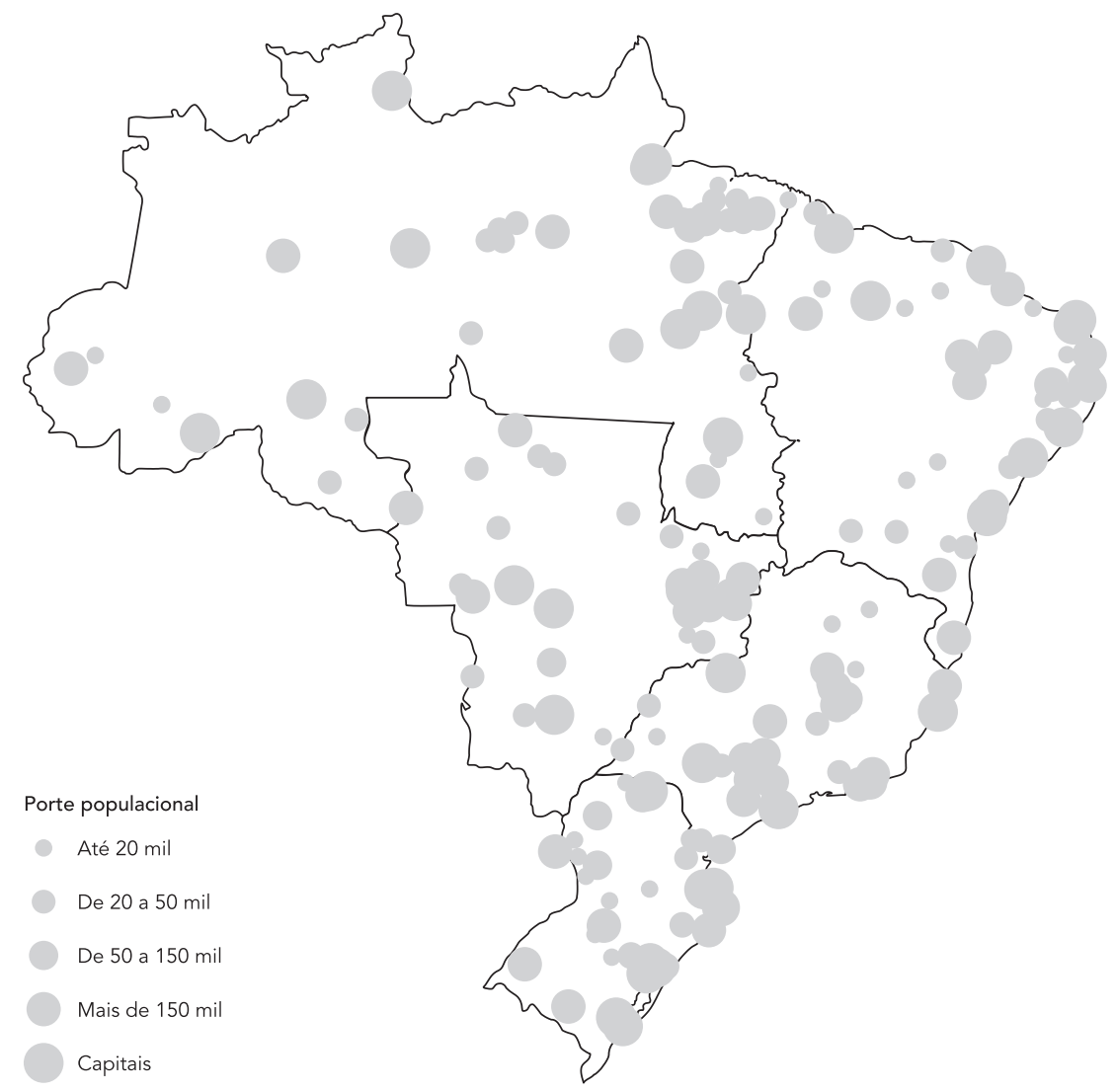

\section{- Sorteio das UPA - Capitais}

Nas capitais, os 30 setores censitários foram sorteados com base em técnica semelhante à anterior. A referência, nesse caso, passou a ser o número de domicílios particulares permanentes urbanos (DOMPPU) em cada setor. De posse da base de dados de setores censitários fornecida pelo IBGE, foram inicialmente eliminados os setores não urbanos e os especiais do tipo quartéis, alojamentos, penitenciárias, hospitais, aldeias, dentre outros (classificados pelo IBGE com códigos 2 a 7).

Utilizando ferramentas de geoprocessamento, um mapa com a identificação dos setores sorteados foi elaborado para cada capital, de modo a observar sua distribuição no território. Os mapas individuais de cada setor foram, então, adquiridos junto ao IBGE de modo a que pudessem municiar o trabalho das equipes de campo.

\section{Número mínimo de entrevistas/exames}

O estudo de precisão considerou os domínios demográficos agrupados segundo o grau de densidade no total da população e a variabilidade interna dos índices de saúde bucal.

Para os grupos etários referidos em anos completos (5, 12 e 65 a 74), adotou-se o coeficiente de variação como indicador de precisão para estimativas de prevalências [expressão (1)]. Escolheu-se o número mínimo de entrevistas 9,10, esperando-se que as prevalências estimadas (P) fossem maiores que $10 \%$ e que seus erros padrão [epa(p)] não ultrapassassem 15\% desses valores.

$c \nu(p)=\left[\frac{e p a(p)}{(p)}\right]=\sqrt{\frac{d^{2} \cdot p \cdot(100-p)}{(n p)}} \leq 15 \%$

Os dados da Tabela 2 mostram resultados para coeficientes de variação segundo diferentes 
valores de prevalências e tamanhos de amostra já corrigidos pelo efeito do delineamento (deff) $=$ 2. Nota-se que os mesmos não ultrapassam $15 \%$ quando $\mathrm{n}=250$ e as prevalências estão acima de $10 \%$ (esperado no projeto). Ou seja, considerando a precisão relativa como critério de confiabilidade, tolera-se que o erro padrão (erro de amostragem) seja, no máximo, igual a $15 \%$ da prevalência estimada.

Nos grupos de 15 a 19 e 35 a 44 anos, calculouse o tamanho (n) da amostra final pela expressão $\mathrm{n}=\left[\left(\mathrm{s}_{\mathrm{x}} \cdot 1,96\right) / \mathrm{m}\right]^{2}$, em que 1,96 é o termo da distribuição normal correspondente ao intervalo de $95 \%$ de confiança, e "m" é a margem tolerada para o erro inerente ao processo de amostragem aleatória simples 11. Estimativas para a variância da média do índice CPO (dentes cariados, perdidos e obturados) ( $\mathrm{s}_{\mathrm{x}}$ ) foram calculadas baseandose nos dados do levantamento anterior, realizado em 200312.

Os resultados iniciais foram corrigidos para compensar o efeito de taxas de respostas em torno de $80 \%$ e efeito de desenho (deff) igual a 2 para proteger o impacto do delineamento por conglomerados sobre a precisão inicialmente fixada, admitindo o processo de amostragem como aleatória simples.

\section{Número de domicílios}

As amostras de domicílios nos 32 domínios geográficos foram calculadas pela expressão (dom = n / r x 0,9), em que "n" é o número mínimo de entrevistas, determinadas pelo critério de precisão, e "r" é a densidade de elementos (de cada grupo demográfico) por domicílio, calculada com base nos dados do censo demográfico de 2000. A correção de 0,9 tem a finalidade de prevenir perdas de precisão devido a domicílios fechados, vagos ou recusas em participar do estudo.

Por exemplo, em determinada capital, para alcançar 250 entrevistas na população de crianças ou de idosos, seria necessário sortear, respectivamente, 5.636 e 1.904 domicílios. Essa diferença resulta das densidades desiguais, calculadas pela razão (indivíduos/domicílio), que resultaram em 5 crianças ou 15 idosos para cada 100 domicílios.

A Tabela 3 ilustra os tamanhos de amostra e os respectivos números de domicílios para cada grupo etário.

Considerando-se os totais de cada domínio, deveriam ser examinados e entrevistados 47.005 indivíduos.

\section{Sorteio dos domicílios e dos elementos amostrais}

Definidos os municípios e o número de indivíduos a ser investigado em cada um deles, e tendo sido sorteados os setores nas capitais e nos municípios do interior, foi estabelecido o estágio seguinte: o sorteio dos domicílios.

Idealmente, o estágio seguinte de sorteio deveria ter seguido a mesma lógica dos anteriores, partindo de uma lista de endereços e realizandose um sorteio sistemático ${ }^{7}$. Ocorre que essa situação nem sempre é possível, pois, assim como os setores censitários são subdivisões razoavelmente estáveis e é possível listá-los e realizar um sorteio, o mesmo não acontece com os endereços. As listas de endereços, quando estão disponíveis, são de difícil atualização.

Optou-se, então, pelo procedimento de arrolamento e sorteio em um mesmo momento. Com base na densidade domiciliar de cada grupo

Tabela 2

Erros padrão (EP) e coeficientes de variação (CV) segundo tamanho de amostra em estudos transversais.

\begin{tabular}{|c|c|c|c|c|c|c|c|c|c|c|}
\hline \multirow[t]{3}{*}{$\mathbf{N}$} & \multicolumn{10}{|c|}{ Prevalências (\%) } \\
\hline & \multicolumn{2}{|c|}{5} & \multicolumn{2}{|c|}{10} & \multicolumn{2}{|c|}{25} & \multicolumn{2}{|c|}{40} & \multicolumn{2}{|c|}{50} \\
\hline & EP & CV (\%) & EP & CV (\%) & EP & CV (\%) & EP & CV (\%) & EP & CV (\%) \\
\hline 250 & 1,95 & 39 & 2,68 & 27 & 3,87 & 15 & 4,38 & 11 & 4,47 & 9 \\
\hline 500 & 1,38 & 28 & 1,90 & 19 & 2,74 & 11 & 3,10 & 8 & 3,16 & 6 \\
\hline 750 & 1,13 & 23 & 1,55 & 15 & 2,24 & 9 & 2,53 & 6 & 2,58 & 5 \\
\hline 1.000 & 0,97 & 19 & 1,34 & 13 & 1,94 & 8 & 2,19 & 5 & 2,24 & 4 \\
\hline 1.500 & 0,80 & 16 & 1,10 & 11 & 1,58 & 6 & 1,79 & 4 & 1,83 & 4 \\
\hline 2.000 & 0,44 & 14 & 0,95 & 9 & 1,37 & 5 & 1,55 & 4 & 1,58 & 3 \\
\hline
\end{tabular}

Nota: limites aceitáveis: $C V \leq 15 \%$ e $E P \leq 3 \%$

Fonte: Organização das Nações Unidas 7 . 
Tabela 3

Tamanhos de amostra e número de domicílios requerido segundo grupo etário e domínio (capital e interior).

\begin{tabular}{|c|c|c|c|c|c|c|c|c|c|c|}
\hline \multirow[t]{3}{*}{ Domínios } & \multicolumn{10}{|c|}{ Idade ou grupo etário (em anos completos de vida) } \\
\hline & \multicolumn{2}{|c|}{5} & \multicolumn{2}{|c|}{12} & \multicolumn{2}{|c|}{15 a 19} & \multicolumn{2}{|c|}{35 a 44} & \multicolumn{2}{|c|}{65 a 74} \\
\hline & $\mathbf{n}$ & Domicílios & $\mathbf{n}$ & Domicílios & $\mathbf{n}$ & Domicílios & $\mathbf{n}$ & Domicílios & $\mathbf{n}$ & Domicílios \\
\hline \multicolumn{11}{|l|}{ Capitais } \\
\hline Porto Velho & 250 & 3.238 & 250 & 3.561 & 200 & 577 & 487 & 999 & 250 & 4.093 \\
\hline Rio Branco & 250 & 3.376 & 250 & 3.441 & 481 & 1.379 & 559 & 1.251 & 250 & 2.884 \\
\hline Manaus & 250 & 3.769 & 250 & 3.408 & 238 & 681 & 553 & 1.151 & 250 & 3.238 \\
\hline Boa Vista & 250 & 3.561 & 250 & 3.653 & 200 & 568 & 390 & 825 & 250 & 2.993 \\
\hline Belém & 250 & 4.397 & 250 & 3.913 & 200 & 626 & 780 & 1.544 & 250 & 2.120 \\
\hline Macapá & 250 & 2.955 & 250 & 3.152 & 200 & 535 & 467 & 1.106 & 250 & 3.769 \\
\hline Palmas & 250 & 3.671 & 250 & 3.238 & 212 & 589 & 443 & 919 & 250 & 5.124 \\
\hline São Luís & 250 & 4.290 & 250 & 4.036 & 200 & 622 & 508 & 1.133 & 250 & 2.855 \\
\hline Teresina & 250 & 4.218 & 250 & 4.290 & 200 & 642 & 813 & 1.842 & 250 & 2.662 \\
\hline Fortaleza & 250 & 4.550 & 250 & 4.148 & 200 & 626 & 668 & 1.428 & 250 & 2.241 \\
\hline Natal & 250 & 4.692 & 250 & 4.416 & 262 & 871 & 390 & 844 & 250 & 2.057 \\
\hline João Pessoa & 250 & 4.972 & 250 & 4.442 & 210 & 679 & 502 & 1.054 & 250 & 2.007 \\
\hline Recife & 250 & 5.250 & 250 & 4.663 & 200 & 699 & 475 & 968 & 250 & 1.734 \\
\hline Maceió & 250 & 3.890 & 250 & 4.036 & 228 & 736 & 502 & 1.088 & 250 & 2.545 \\
\hline Aracaju & 250 & 4.496 & 250 & 4.550 & 200 & 694 & 505 & 1.074 & 250 & 2.145 \\
\hline Salvador & 250 & 4.782 & 250 & 4.692 & 200 & 713 & 398 & 815 & 250 & 2.368 \\
\hline Belo Horizonte & 250 & 6.156 & 250 & 5.865 & 200 & 890 & 457 & 1.099 & 250 & 1.812 \\
\hline Vitória & 250 & 6.113 & 250 & 5.987 & 200 & 913 & 476 & 1.181 & 250 & 1.920 \\
\hline Rio de Janeiro & 250 & 6.028 & 250 & 6.028 & 200 & 966 & 411 & 1.014 & 250 & 1.489 \\
\hline São Paulo & 250 & 5.637 & 250 & 5.749 & 200 & 913 & 415 & 970 & 250 & 1.904 \\
\hline Curitiba & 250 & 6.493 & 250 & 5.781 & 204 & 882 & 480 & 1.122 & 250 & 2.113 \\
\hline Florianópolis & 250 & 6.840 & 250 & 6.137 & 200 & 897 & 307 & 742 & 250 & 2.036 \\
\hline Porto Alegre & 250 & 6.737 & 250 & 6.096 & 200 & 951 & 321 & 853 & 250 & 1.572 \\
\hline Campo Grande & 250 & 5.425 & 250 & 5.229 & 200 & 779 & 469 & 1.139 & 250 & 2.237 \\
\hline Cuiabá & 250 & 5.198 & 250 & 5.325 & 200 & 781 & 427 & 1.029 & 250 & 2.738 \\
\hline Goiânia & 250 & 5.826 & 250 & 5.564 & 253 & 1.062 & 375 & 872 & 250 & 2.309 \\
\hline Brasília & 250 & 5.106 & 250 & 4.960 & 200 & 772 & 526 & 1.157 & 250 & 2.913 \\
\hline \multicolumn{11}{|l|}{ Interior/Região } \\
\hline Norte & 250 & 3.073 & 250 & 3.289 & 214 & 577 & 597 & 1.506 & 250 & 2.261 \\
\hline Nordeste & 250 & 3.610 & 250 & 3.940 & 235 & 731 & 618 & 1.634 & 250 & 1.695 \\
\hline Sudeste & 250 & 5.306 & 250 & 5.413 & 211 & 883 & 581 & 1.485 & 250 & 1.623 \\
\hline Sul & 250 & 6.021 & 250 & 5.605 & 208 & 892 & 546 & 1.406 & 250 & 1.509 \\
\hline Centro-oeste & 250 & 4.896 & 250 & 5.076 & 256 & 1.000 & 547 & 1.336 & 250 & 1.955 \\
\hline
\end{tabular}

etário e do total de domicílios em cada setor foram calculadas as frações de domicílios, as quais indicavam quais domicílios deveriam ser pesquisados em função da probabilidade de ocorrência de moradores nos grupos etários elegíveis. As frações para cada grupo etário foram, então, colocadas em folhas de arrolamento e sorteio de modo que, em cada domicílio, havia a indicação precisa para busca das respectivas unidades elegíveis nos diversos grupos etários (ver exemplo na Figura 3).
Entretanto, a confecção da folha de arrolamento e sorteio de domicílios exigia que a informação do número de DOMPPU fosse a mais atualizada possível, pois este era um fator determinante para o estabelecimento dos intervalos de amostragem em cada grupo etário.

O número de DOMPPU constante na base de dados dos setores tinha origens e graus de atualização diferentes. Como se sabe, o IBGE realiza a cada 10 anos o censo demográfico e é nestas ocasiões que toda sua base de setores é atuali- 


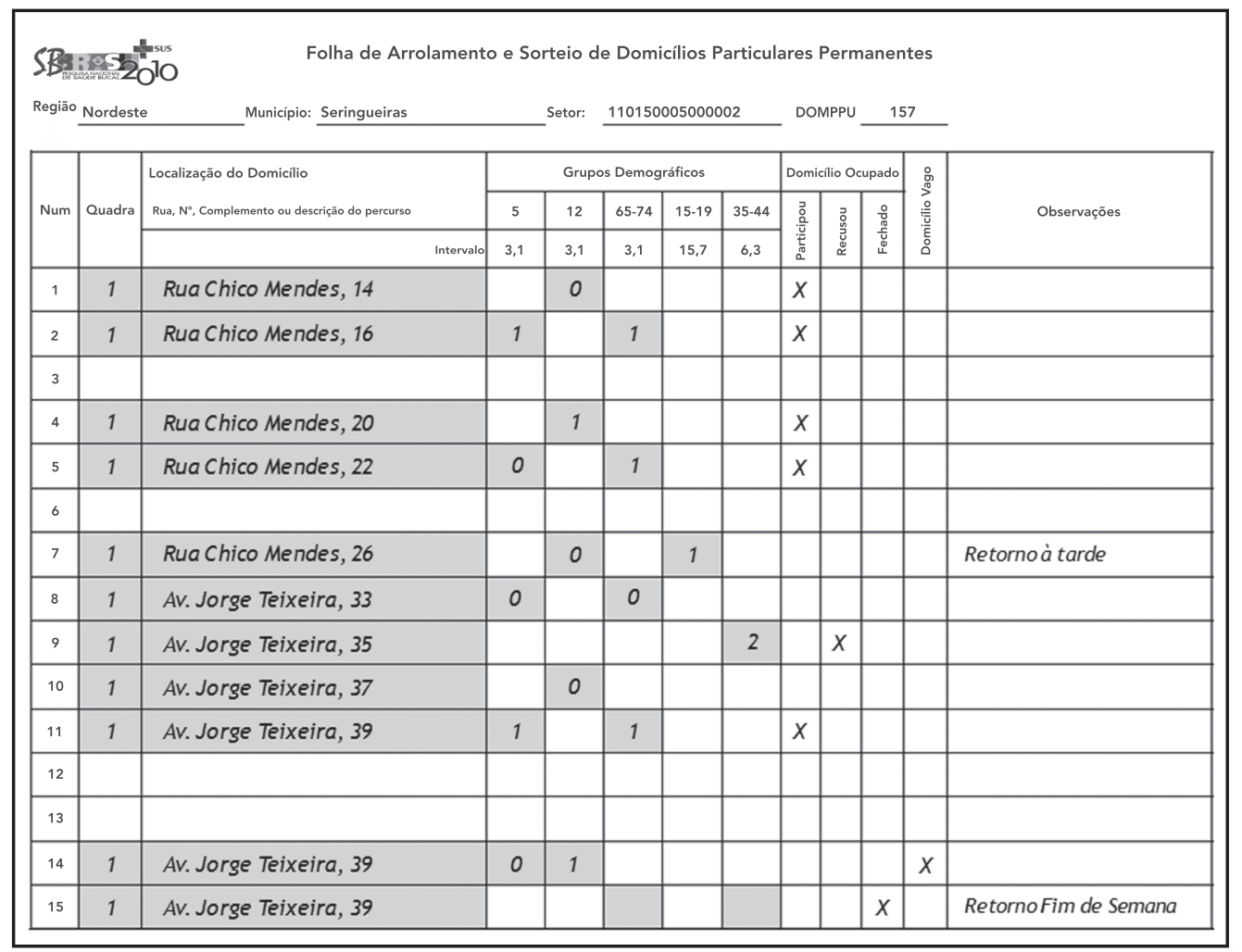

zada. Entre os censos são realizadas as Pesquisas Nacionais por Amostra de Domicílios (PNAD) e também outros estudos específicos. Em 2007, o IBGE realizou a Contagem Populacional em 5.435 municípios, a grande maioria com menos de 150 mil habitantes. Nos outros 128 municípios (os quais incluem grande parte das capitais e municípios de grande porte) não foi realizada a contagem populacional, portanto a última informação disponível era a do ano do último censo (2000).

Desse modo, nos municípios sorteados para comporem a amostra do SBB10 havia aqueles em que sua base de setores era de 2007 (portanto razoavelmente atualizada) e aqueles cuja base era do censo 2000, com quase uma década de defasagem. Em decorrência, foram definidas estratégias distintas para estes dois tipos de município. Desse modo, optou-se para os que tinham a base de 2000, para realizar uma atividade prévia (descrita adiante) a fim de corrigir a defasagem no número de domicílios em cada setor censitário 13 .
A Tabela 4 mostra a distribuição dos municípios da amostra do SBB10 de acordo com o ano da base cartográfica de setores. Nos municípios de base cartográfica 2007, foi utilizada, para a elaboração das folhas de arrolamento, a informação disponível na base de dados do IBGE. Já para as capitais e municípios com base cartográfica de 2000 foi realizada uma contagem rápida de domicílios em cada um dos setores sorteados. O número obtido nessa contagem foi utilizado como referência para a construção das folhas de arrolamento. O detalhamento de todo o processo de contagem rápida e posterior elaboração das folhas para a atividade de campo pode ser encontrado no Manual da Equipe de Campo, uma das publicações elaboradas para dar suporte às atividades das equipes e está disponível no sítio do projeto (http:/ /www.sbbrasil2010.org ou http://www.saude.gov.br/bucal). 
Municípios da amostra do SBB10 de acordo com o domínio e o ano da base cartográfica dos setores censitários.

\begin{tabular}{|c|c|c|}
\hline Região & UF & Município \\
\hline \multicolumn{3}{|c|}{ Capitais e base cartográfica 2000} \\
\hline \multirow[t]{9}{*}{ Norte } & $\mathrm{RO}$ & 110020 Porto Velho \\
\hline & $A C$ & 120040 Rio Branco \\
\hline & AM & 130260 Manaus \\
\hline & $\mathrm{RR}$ & 140010 Boa Vista \\
\hline & PA & 150080 Ananindeua \\
\hline & PA & 150140 Belém \\
\hline & PA & 150680 Santarém \\
\hline & AP & 160030 Macapá \\
\hline & TO & 172100 Palmas \\
\hline \multirow[t]{12}{*}{ Nordeste } & MA & 211130 São Luís \\
\hline & $\mathrm{Pl}$ & 221100 Teresina \\
\hline & CE & 230440 Fortaleza \\
\hline & CE & 230730 Juazeiro do Norte \\
\hline & $\mathrm{RN}$ & 240810 Natal \\
\hline & PB & 250750 João Pessoa \\
\hline & PE & 260410 Caruaru \\
\hline & PE & 261160 Recife \\
\hline & $\mathrm{AL}$ & 270430 Maceió \\
\hline & SE & 280030 Aracaju \\
\hline & BA & 290570 Camaçari \\
\hline & BA & 292740 Salvador \\
\hline \multirow[t]{16}{*}{ Sudeste } & MG & 310620 Belo Horizonte \\
\hline & MG & 310670 Betim \\
\hline & MG & 316720 Sete Lagoas \\
\hline & MG & 317020 Uberlândia \\
\hline & ES & 320530 Vitória \\
\hline & RJ & 330170 Duque de Caxias \\
\hline & RJ & 330330 Niterói \\
\hline & RJ & 330455 Rio de Janeiro \\
\hline & SP & 350600 Bauru \\
\hline & SP & 350950 Campinas \\
\hline & SP & 351870 Guarujá \\
\hline & SP & 353440 Osasco \\
\hline & SP & 354390 Rio Claro \\
\hline & SP & 354850 Santos \\
\hline & SP & 355030 São Paulo \\
\hline & SP & 355280 Taboão da Serra \\
\hline \multirow[t]{11}{*}{ Sul } & PR & 410690 Curitiba \\
\hline & PR & 410830 Foz do Iguaçu \\
\hline & PR & 411370 Londrina \\
\hline & PR & 412550 São José dos Pinhais \\
\hline & SC & 420240 Blumenau \\
\hline & $\mathrm{SC}$ & 420540 Florianópolis \\
\hline & RS & 430920 Gravataí \\
\hline & RS & 431340 Novo Hamburgo \\
\hline & RS & 431440 Pelotas \\
\hline & RS & 431490 Porto Alegre \\
\hline & RS & 431560 Rio Grande \\
\hline
\end{tabular}

(continua) 
Tabela 4 (continuação)

\begin{tabular}{|c|c|c|}
\hline Região & UF & Município \\
\hline \multirow[t]{7}{*}{ Centro-oeste } & MS & 500270 Campo Grande \\
\hline & MT & 510340 Cuiabá \\
\hline & GO & 520110 Anápolis \\
\hline & GO & 520140 Aparecida de Goiânia \\
\hline & GO & 520870 Goiânia \\
\hline & GO & 521250 Luziânia \\
\hline & DF & 530010 Brasília \\
\hline \multicolumn{3}{|c|}{ Interior e base cartográfica 2007} \\
\hline \multirow[t]{28}{*}{ Norte } & $\mathrm{RO}$ & 110013 Machadinho d'Oeste \\
\hline & $\mathrm{RO}$ & 110030 Vilhena \\
\hline & $\mathrm{RO}$ & 110150 Seringueiras \\
\hline & $A C$ & 120020 Cruzeiro do Sul \\
\hline & $A C$ & 120034 Manoel Urbano \\
\hline & $A M$ & 130050 Barreirinha \\
\hline & AM & 130180 Ipixuna \\
\hline & AM & 130185 Iranduba \\
\hline & AM & 130340 Parintins \\
\hline & AM & 130420 Tefé \\
\hline & PA & 150180 Breves \\
\hline & PA & 150210 Cametá \\
\hline & PA & 150230 Capitão Poço \\
\hline & PA & 150275 Concórdia do Pará \\
\hline & PA & 150330 Igarapé-Mirim \\
\hline & PA & 150375 Jacareacanga \\
\hline & PA & 150390 Juruti \\
\hline & PA & 150405 Mãe do Rio \\
\hline & PA & 150520 Oeiras do Pará \\
\hline & PA & 150553 Parauapebas \\
\hline & PA & 150618 Rondon do Pará \\
\hline & PA & 150730 São Félix do Xingu \\
\hline & PA & 150740 São Francisco do Pará \\
\hline & PA & 150810 Tucuruí \\
\hline & $A P$ & 160060 Santana \\
\hline & TO & 170370 Brejinho de Nazaré \\
\hline & TO & 170900 Goiatins \\
\hline & TO & 170950 Gurupi \\
\hline \multirow[t]{15}{*}{ Nordeste } & MA & 210370 Cururupu \\
\hline & MA & 210530 Imperatriz \\
\hline & MA & 210620 Luís Domingues \\
\hline & MA & 211223 Trizidela do Vale \\
\hline & $\mathrm{Pl}$ & 220260 Castelo do Piauí \\
\hline & CE & 230110 Aracati \\
\hline & CE & 230365 Catunda \\
\hline & CE & 230780 Marco \\
\hline & CE & 230810 Mauriti \\
\hline & RN & 240070 Alto do Rodrigues \\
\hline & RN & 240325 Parnamirim \\
\hline & PB & 250050 Alagoinha \\
\hline & PB & 251620 Sousa \\
\hline & PE & 260005 Abreu e Lima \\
\hline & PE & 260590 Gameleira \\
\hline
\end{tabular}

(continua) 
Tabela 4 (continuação)

\begin{tabular}{|c|c|c|}
\hline Região & UF & Município \\
\hline \multirow[t]{12}{*}{ Nordeste } & PE & 260830 Jupi \\
\hline & PE & 261220 Salgueiro \\
\hline & $\mathrm{AL}$ & 270940 Viçosa \\
\hline & SE & 280040 Arauá \\
\hline & BA & 290060 Aiquara \\
\hline & BA & 290530 Cafarnaum \\
\hline & BA & 290550 Caldeirão Grande \\
\hline & BA & 291270 Ibirapitanga \\
\hline & BA & 291640 Itapetinga \\
\hline & BA & 291950 Livramento de Nossa Senhora \\
\hline & BA & 293015 Serra do Ramalho \\
\hline & BA & 293135 Teixeira de Freitas \\
\hline \multirow[t]{18}{*}{ Sudeste } & MG & 310240 Alvorada de Minas \\
\hline & MG & 311650 Claro dos Poções \\
\hline & MG & 312090 Curvelo \\
\hline & MG & 312780 Grão Mogol \\
\hline & MG & 314560 Oliveira \\
\hline & MG & 314740 Paraopeba \\
\hline & MG & 314790 Passos \\
\hline & ES & 320320 Linhares \\
\hline & RJ & 330270 Maricá \\
\hline & RJ & 330395 Pinheiral \\
\hline & SP & 350620 Bento de Abreu \\
\hline & $\mathrm{SP}$ & 350760 Bragança Paulista \\
\hline & $\mathrm{SP}$ & 351280 Cosmópolis \\
\hline & SP & 351410 Dois Córregos \\
\hline & SP & 351515 Engenheiro Coelho \\
\hline & SP & 354130 Presidente Epitácio \\
\hline & SP & 354330 Ribeirão Pires \\
\hline & $\mathrm{SP}$ & 354910 São João Boa Vista \\
\hline \multirow[t]{22}{*}{ Sul } & PR & 410150 Arapongas \\
\hline & PR & 410840 Francisco Beltrão \\
\hline & PR & 410860 Goioerê \\
\hline & PR & 411320 Lapa \\
\hline & PR & 411360 Lobato \\
\hline & PR & 411980 Planalto \\
\hline & PR & 412080 Quatro Barras \\
\hline & PR & 412855 Vera Cruz do Oeste \\
\hline & SC & 420820 Itajaí \\
\hline & SC & 420940 Laguna \\
\hline & SC & 421440 Rio das Antas \\
\hline & SC & 421650 São Joaquim \\
\hline & SC & 421670 São José do Cedro \\
\hline & SC & 421870 Tubarão \\
\hline & RS & 430040 Alegrete \\
\hline & RS & 430160 Bagé \\
\hline & RS & 430470 Carazinho \\
\hline & RS & 430720 Erval Grande \\
\hline & RS & 431000 Ibirubá \\
\hline & RS & 431760 Santo Antônio da Patrulha \\
\hline & RS & 432067 Sinimbu \\
\hline & RS & 432145 Teutônia \\
\hline
\end{tabular}

(continua) 
Tabela 4 (continuação)

\begin{tabular}{|c|c|c|}
\hline Região & UF & Município \\
\hline \multirow[t]{27}{*}{ Centro-oeste } & MS & 500100 Aparecida do Taboado \\
\hline & MS & 500110 Aquidauana \\
\hline & MS & 500320 Corumbá \\
\hline & MS & 500330 Coxim \\
\hline & MS & 500755 Santa Rita do Pardo \\
\hline & MS & 500800 Terenos \\
\hline & MT & 510025 Alta Floresta \\
\hline & MT & 510250 Cáceres \\
\hline & MT & 510510 Juara \\
\hline & MT & 510558 Marcelândia \\
\hline & MT & 510562 Mirassol d'Oeste \\
\hline & MT & 510619 Nova Santa Helena \\
\hline & MT & 510718 Ribeirão Cascalheira \\
\hline & MT & 510730 São José Rio Claro \\
\hline & MT & 510840 Várzea Grande \\
\hline & GO & 520470 Campinorte \\
\hline & GO & 520490 Campos Belos \\
\hline & $\mathrm{GO}$ & 520735 Edealina \\
\hline & $\mathrm{GO}$ & 520860 Goianésia \\
\hline & $\mathrm{GO}$ & 521040 ltaberaí \\
\hline & $\mathrm{GO}$ & 521120 Itapuranga \\
\hline & $\mathrm{GO}$ & 521380 Morrinhos \\
\hline & GO & 521405 Mundo Novo \\
\hline & $\mathrm{GO}$ & 521523 Novo Gama \\
\hline & GO & 521760 Planaltina \\
\hline & $\mathrm{GO}$ & 522140 Trindade \\
\hline & GO & 522170 Uruana \\
\hline
\end{tabular}

Unidades da Federação: AC (Acre), AL (Alagoas), AM (Amazonas), AP (Amapá), BA (Bahia), CE (Ceará), DF (Distrito Federal), ES (Espírito Santo), GO (Goiás), MA (Maranhão), MG (Minas Gerais), MS (Mato Grosso do Sul), MT (Mato Grosso), PA (Pará), PB (Paraíba), PE (Pernambuco), PI (Piauí), PR (Paraná), RJ (Rio de Janeiro), RN (Rio Grande do Norte), RO (Rondônia), RR (Roraima), RS (Rio Grande do Sul), SC (Santa Catarina), SE (Sergipe), SP (São Paulo), TO (Tocantins).

\section{Condições pesquisadas}

A manutenção de uma base metodológica uniforme é um requisito importante quando se considera a realização de estudos seccionais como um componente de destaque nas estratégias de vigilância da saúde bucal. No caso particular das doenças e agravos bucais, em sua maioria não são possíveis, nem convenientes, avaliações a partir da mera aferição da prevalência, estabelecida pelo diagnóstico, no indivíduo, da presença ou ausência de doença.

Historicamente, têm sido desenvolvidos diversos índices voltados para a análise das condições bucais mais prevalentes (cárie, doença periodontal, oclusopatias, fluorose, dentre outras), no sentido de se verificar, além da prevalência, a extensão da gravidade ou magnitude dos agravos bucais. A iniciativa da OMS permitiu um amplo, progressivo e consistente aperfeiçoamento dessas ferramentas de investigação de modo que, atualmente, a grande maioria das pesquisas realizadas mundialmente segue um padrão semelhante com relação aos índices utilizados.

Por esse motivo, os índices utilizados no SBB10 e os acréscimos ou modificações atendem às recomendações da OMS na 4 a edição de seu Manual de Instruções para Levantamento Epidemiológico Básico em Saúde Bucal 5, e levam em conta a experiência acumulada no Brasil, em várias regiões, notadamente a partir dos anos 1980 .

Além dos índices tradicionais para aferição dos agravos bucais, também foi aplicado, aos indivíduos examinados, um questionário contendo questões relativas à caracterização socioeconômica, utilização de serviços odontológicos e morbidade bucal autorreferida e autopercepção de saúde bucal. 
Apresentam-se a seguir, de modo resumido, as condições observadas pelos examinadores do SBB10, os índices adotados e algumas alterações propostas para o estudo. Maiores detalhes sobre os códigos, critérios e técnicas de aplicação dos índices podem ser obtidos nos manuais técnicos elaborados para subsidiar as equipes de campo e coordenação, e disponíveis no já citado sítio eletrônico do projeto.

\section{Cárie dentária}

A despeito de seu acentuado declínio em crianças e adolescentes, observado tanto em termos mundiais como nacional, a cárie dentária continua sendo o principal problema de saúde bucal a ser enfrentado no Brasil. Em adultos e idosos, embora a presença de lesões ativas seja menos frequente, as sequelas da doença aparecem como a principal característica a ser avaliada 3 .

Desde o final da década de 1930, quando foi proposto pela primeira vez o índice CPO-D (contagem de dentes cariados, perdidos e obturados), a cárie dentária vem sendo analisada a partir da mensuração dos dentes afetados. Em seus mais de 70 anos de uso rotineiro em pesquisas epidemiológicas, o CPO-D vem sofrendo constantes atualizações pela OMS, desde a primeira edição do Oral Health Surveys: Basic Methods em 1977. Na mais recente edição, a quarta, utilizada como referência neste estudo, foram acrescentadas a avaliação das necessidades de tratamento, o diagnóstico da cárie de raiz, além de outras mudanças nos códigos e critérios, embora a base teórica do índice continue a mesma 5 .

Desse modo, foi utilizado o índice preconizado pela OMS, do qual se pode inferir o CPO-D médio (dentição permanente) e o ceo-d (dentição decídua). Por meio do registro das necessidades de tratamento, pôde-se identificar, além das necessidades propriamente ditas, a presença de lesões não cavitadas (mancha branca presente) e os diferentes níveis da doença ativa (cárie de esmalte, cárie de dentina e cárie próxima à polpa). Portanto, uma maior qualificação do índice pode ser proporcionada pela combinação das distintas medidas de necessidades de tratamento.

\section{Condição periodontal}

O índice mais utilizado em inquéritos populacionais para a aferição da condição periodontal tem sido o CPI (Índice Periodontal Comunitário), proposto pela OMS 14, e complementado pelo exame da Perda de Inserção Periodontal (PIP) para população adulta e idosa. O CPI verifica a ocorrência de sangramento, cálculo e presença de bolsa periodontal (rasa e profunda), tendo co- mo referência o exame por sextante (grupos de 6 dentes entre os 32 da arcada dentária).

Especificamente com relação ao CPI, o modo de aferição foi modificado no sentido de obter a prevalência individualizada dos agravos (sangramento, cálculo e bolsa). Tratou-se de uma estratégia importante, pois o CPI tradicional, ao referir apenas o pior escore do sextante, em geral tende a mascarar a real prevalência desses agravos.

\section{Traumatismo dentário}

Embora na aferição da condição dentária os dentes que apresentem lesões traumáticas sejam codificados (código “T” do CPO), há uma nítida perda de informação, particularmente por dois aspectos. Em primeiro lugar, nos casos em que há uma lesão de cárie associada perde-se a informação do trauma, uma vez que prevalece a informação de cárie dentária. Em segundo, a informação é demasiadamente simplificada, podendo uma pequena fratura ser codificada do mesmo modo que uma perda de estrutura dentária de maiores proporções. Além disso, não é possível saber quando o dente é perdido por trauma, pois o mesmo código é usado para perdas por outros motivos.

Desse modo, julgou-se importante que o traumatismo dentário fosse avaliado como uma medida específica, em separado. Para tanto, foram utilizados os critérios que indicavam sinais de fratura coronária e avulsão dentária. Para esse exame foram considerados os incisivos superiores e inferiores permanentes.

\section{Oclusão dentária}

A primeira proposta da OMS para avaliar condições populacionais de oclusão dentária apareceu em 1987, na terceira edição do Oral Health Surveys: Basic Methods. O índice propunha uma classificação bastante simplória das condições de cada indivíduo, considerando a presença e o grau de alguma oclusopatia, com a seguinte codificação: 0-nenhuma oclusopatia; 1 - oclusopatia leve; e 2 - oclusopatia moderada ou grave 15.

Esse modelo, apesar de apontar para uma possibilidade de padronização em termos mundiais, era muito limitado, subjetivo e excessivamente simplificado. Em sua quarta edição, o manual da OMS propôs um novo índice de avaliação de oclusopatias, proposto anos antes por Cons et al. 16, chamado DAI (Índice de Estética Dental - sigla derivada da expressão inglesa Dental Aesthetic Index). O princípio básico do DAI é de uma combinação de medidas (não somente de problemas oclusais) as quais, em seu conjunto, expressam o estado oclusal do indivíduo e, con- 
sequentemente, sua necessidade de tratamento, devido à composição do índice que considera comprometimento estético além da oclusão. Ao todo são obtidas 11 medidas, considerando-se três grandes dimensões a serem avaliadas: a dentição, o espaço e a oclusão propriamente dita.

Desse modo, no inquérito populacional SBB10 o DAI foi utilizado para avaliação das anormalidades dentofaciais, na idade de 12 anos e na faixa etária de 15 a 19 anos.

Por outro lado, têm sido publicados estudos epidemiológicos incluindo a avaliação da oclusão na dentição decídua, o que pode ser interpretado como manifestação de interesse, no nível mundial, em medidas dessas condições na infância. Contudo, o DAI permite avaliar os problemas oclusais somente na dentição permanente e, por este motivo, no SBB10 a oclusão na dentição decídua foi avaliada com o emprego do Índice de Foster \& Hamilton 17.

\section{Fluorose dentária}

A fluorose é caracterizada como um distúrbio específico de formação do dente, com alterações na estrutura do esmalte dentário, causadas pela ingestão crônica e excessiva de flúor durante período de formação da dentição. A manifestação clínica depende da quantidade ingerida, da duração de exposição, da idade e da susceptibilidade individual 18 .

Pela necessidade de acompanhar os efeitos do uso de medidas de saúde pública de amplo impacto como a fluoretação das águas, o uso de creme dental fluoretado, programas preventivos de aplicação tópica de flúor e suas implicações na saúde da população, considerou-se necessário incluir no SBB10 a observação da fluorose.

Dean, em 1934, desenvolveu a primeira classificação de fluorose dentária sendo mais tarde modificada por ele. A classificação conhecida como Índice de Dean 19 tem sido usada por muitos anos para descrever a fluorose, o que permite a comparação com um volume maior de estudos. É o índice recomendado pela OMS para estudos de fluorose dentária em populações. Além disso, dada a alta subjetividade envolvida na aferição dessa condição, é o instrumento epidemiológico de escolha para inquéritos populacionais, tendo em vista a obtenção de melhores níveis de reprodutibilidade em relação a outros índices.

\section{Edentulismo}

A inclusão do uso e das necessidades de prótese na população, neste projeto, atende a três indicações: seguem a orientação da OMS para levantamentos epidemiológicos, permite a comparação histórica e atende às necessidades de planejamento específicas desta área, particularmente quando se considera um dos eixos da Política Nacional de Saúde Bucal, relativo à atenção secundária por intermédio dos Centros de Especialidades Odontológicas (CEO).

Na prática, a avaliação do uso e necessidade de prótese ajuda a entender o agravo "edentulismo", servindo, ao mesmo tempo, para estimar a gravidade do problema pela análise conjunta dos dados de uso e necessidade, e para subsidiar ações de planejamento baseando-se na análise das necessidades.

Um aspecto para o qual cabe destaque é que a verificação da necessidade de prótese deve incluir uma avaliação da sua qualidade quando a prótese está presente. Os dois índices não são excludentes, ou seja, é possível estar usando e também necessitar de uma prótese. Para uniformizar a avaliação dessas condições, fixou-se um conjunto de critérios de decisão, para calibrar os examinadores. Desse modo, ficou estabelecido que, para determinar se uma prótese que está em uso é inadequada e, portanto, deve ser substituída, seriam observadas as seguintes condições 20: (a) retenção - está folgada ou apertada; (b) estabilidade e reciprocidade - apresenta deslocamento ou báscula; (c) fixação - lesiona os tecidos; e (d) estética - apresenta manchas ou fraturas e não está adequada ao perfil facial do paciente.

Quando pelo menos uma dessas condições estava presente, recomendava-se a substituição da prótese, considerando-se, portanto, haver necessidade de prótese.

\section{Condição socioeconômica, utilização de serviços odontológicos e autopercepção de saúde bucal}

Variáveis relativas a condições subjetivas, importantes para compreender como o processo saúde/doença bucal é percebido no nível individual, foram incluídas no questionário aplicado aos participantes do estudo. Também a condição socioeconômica e o grau de utilização de serviços foi aferido, buscando-se contribuir para o processo de planejamento e organização da rede de atenção à saúde bucal.

O questionário constou de três blocos: (a) caracterização demográfica e socioeconômica; (b) utilização de serviços odontológicos e morbidade bucal referida; e (c) autopercepção e impactos em saúde bucal. Cada um dos blocos era composto por perguntas que foram aplicadas ao responsável pelo domicílio, para obtenção de informações relativas à família e ao próprio indivíduo examinado. 


\section{Treinamento e preparação das equipes}

As equipes de campo, formadas por um examinador e um anotador, foram treinadas em oficinas de trabalho com duração de 32 horas, com o objetivo de discutir a operacionalização das etapas do trabalho, compreender as atribuições de cada participante e assegurar um grau aceitável de uniformidade nos procedimentos. Em cada oficina de treinamento participaram até 10 equipes ao mesmo tempo. As capitais contaram com 10 equipes de campo e os municípios do interior com 2 a 6 equipes, dependendo do porte populacional.

A calibração envolveu pelo menos 8 períodos de 4 horas de trabalho, contemplando os aspectos teóricos e práticos dos índices a serem utilizados. Os procedimentos de calibração foram planejados de modo a antecipar (simular) as condições que os examinadores encontrariam, sobretudo em relação aos diferentes grupos populacionais. Quanto à técnica de calibração, adotou-se a do consenso 21 , calculando-se os coeficientes de concordância entre cada examinador e os resultados obtidos pelo consenso da equipe. Tomou-se como referência o modelo proposto pela OMS 22 e foi calculado o coeficiente kappa ponderado para cada examinador, grupo etário e agravo estudado, tendo como limite mínimo aceitável o valor de 0,65 . Todo o detalhamento da técnica de treinamento e calibração encontra-se disponível no Manual de Calibração de Examinadores no sítio do projeto.

\section{Apuração e análise}

Os dados foram coletados com o emprego de um dispositivo eletrônico (Personal Digital Assistant - PDA), os quais foram cedidos para o SBB10 pelo IBGE para uso durante o trabalho de campo. Foi desenvolvido um software específico para a entrada de dados e cada equipe tinha um PDA disponível. Desse modo, o uso de fichas em papel ocorreu somente em situações excepcionais e apenas como alternativa ao sistema do PDA.

Com relação à análise dos dados, considerando-se que trata-se de amostra complexa, as estimativas de médias, prevalências e respectivos erros padrão foram calculadas com o uso do módulo "Complex Samples" do programa SPSS (SPSS Inc., Chicago, Estados Unidos), que considera as variáveis de planejamento e inclusão dos pesos básicos resultantes do processo de amostragem.

\section{Implicações éticas}

Em acordo com a Resolução $n^{\circ}$. 196/96 do Conselho Nacional de Saúde (CNS), relativa às pesquisas em seres humanos, o SBB2010 foi submetido ao Comitê de Ética em Pesquisa do Ministério da Saúde, foi aprovado e recebeu registro na Comissão Nacional de Ética em Pesquisa (CONEP), do CNS, sob o número 15.498. Cópia do Projeto, do Termo de Consentimento Livre e Esclarecido (TCLE) utilizado e do parecer do CONEP estão disponíveis no sítio eletrônico do projeto.

\section{Considerações finais}

A metodologia apresentada neste artigo foi posta em prática na execução do SBB10, entre o final de 2009 e o final de 2010. A coleta de dados foi concluída e os resultados preliminares já foram anunciados no final de 2010. Foram examinados e entrevistados em seus domicílios mais de 37 mil indivíduos, significando uma taxa geral de resposta de $80 \%$.

Analisando alguns pontos relevantes da aplicação do método, podem ser destacados aspectos positivos e outros que ainda se apresentam como desafios a serem considerados em experiências futuras. Dentre os pontos positivos podem ser destacados principalmente aqueles que geraram aprimoramentos em relação ao SBB03, como, por exemplo, o uso da tecnologia de entrada de dados eletrônica. Em nível nacional, foi a primeira vez que utilizou-se essa técnica em um inquérito de saúde bucal, e foram percebidos ganhos importantes principalmente na segurança e na qualidade dos dados produzidos e na rapidez com que o banco de dados foi elaborado. $\mathrm{O}$ software desenvolvido para a entrada de dados, em ambiente Windows Mobile, é de domínio público e pode ser considerado um produto importante na medida em que poderá ser utilizado livremente em outras iniciativas que tenham a mesma base metodológica.

Outro aspecto relevante diz respeito ao desenho amostral que, mesmo considerando as complexas características demográficas do território brasileiro, conseguiu compor uma amostra com importante poder de precisão. Embora tenha se pautado em uma base já estabelecida nacional e internacionalmente, o plano amostral apresenta características bastante peculiares que podem contribuir de modo significativo para futuros estudos seccionais de outros agravos.

Com relação ao modelo operacional, um ganho importante se deu pelo fato de as equipes de campo terem sido formadas por profissionais do SUS. Em cada município da amostra 
estabeleceu-se uma importante parceria entre as Secretarias Estaduais e a Secretaria Municipal de Saúde, no sentido de capacitar e dar apoio logístico às equipes de campo formadas por Cirurgiões-dentistas (CD) e Auxiliares de Saúde Bucal (ASB) pertencentes à rede de serviços. Desse modo, além de proporcionar o estabelecimento de vínculo do serviço com a pesquisa, as equipes foram capacitadas e, em sua maioria, está hoje preparada para reproduzir estudos semelhantes em seus locais de atuação. Além dos CD e ASB, na maioria dos municípios ocorreu o envolvimento direto dos Agentes Comunitários de Saúde (ACS) apoiando, tanto no processo de contagem de domicílios (descrito anteriormente) quanto no momento da coleta de dados nos domicílios.

Há outros aspectos que também representaram avanços em relação às experiências anteriores, mas que ainda se apresentam como desafios importantes em termos operacionais. A estratégia de treinamento e calibração, por exemplo, teve de dar conta de manter um mesmo padrão de diagnóstico epidemiológico em aproximadamente mil equipes que trabalharam nos $177 \mathrm{mu}$ nicípios da amostra. Esses examinadores tinham de estar calibrados para vários índices de agravos bucais, e ainda ter de dominar as técnicas de percurso e as regras de decisão para o trabalho de campo.

Assim, do ponto de vista de sua aplicabilidade, o método se mostrou viável para utilização em pesquisas de nível nacional em saúde bucal, podendo ser mantido enquanto procedimento padrão para a produção de dados primários dentro de uma estratégia de vigilância em saúde bucal nos diferentes níveis de gestão.

Todo o material necessário para a realização de uma pesquisa nos mesmos moldes é de domínio público e está disponível no sítio do projeto.

\section{Resumo}

O Projeto SBBrasil 2010 (SBB10) foi concebido como um levantamento epidemiológico em saúde bucal, de base nacional, dentro da estratégia de vigilância em saúde. O objetivo deste artigo é apresentar aspectos da metodologia do SBB10 que possam contribuir para ampliar e desenvolver conhecimentos na área de saúde. Com relação ao plano amostral, trata-se de uma pesquisa por conglomerados e com múltiplos estágios. Capitais e municípios do interior das cinco regiões brasileiras compõem os domínios da amostra, cujas unidades amostrais foram, respectivamente, setor censitário e domicílio para as capitais, e município, setor censitário e domicílio para o interior. Nas capitais foram sorteados 30 setores e, no interior de cada região, 30 municípios. A precisão considerou os domínios agrupados segundo o grau de densidade no total da população e a variabilidade interna dos índices. Foram avaliadas as condições de cárie dentária, doença periodontal, oclusopatias, fluorose, traumatismo dentário e edentulismo em cinco grupos etários (5, 12, 15 a 19, 35 a 44 e 65 a 74 anos).

Saúde Bucal; Vigilância Epidemiológica; Estudos Transversais

\section{Colaboradores}

A. G. Roncalli coordenou o projeto, participou da construção do plano amostral e contribuiu na redação do texto. S. J. Moysés, R. A. A. Marques, R. S. Pinto, P. S. A. Goes, N. Figueiredo, M. Paludetto Júnior, M. V. Vettore, M. I. S. Cortes, M. C. M. Freire, M. A. Peres, L. Moura, K. G. Peres, E. Casotti, C. H. S. M. Freitas, A. C. Nascimento e S. C. G. B. Reis participaram da concepção geral do projeto e redação do texto. P. C. Narvai participou da concepção geral do projeto, colaboração no plano amostral e redação do artigo. N. N. Silva foi responsável pelo processo de amostragem e contribuiu na redação do texto. 


\section{Outras instituições}

${ }^{9}$ Faculdade de Ondontologia, Universidade Federal de Goiás, Goiânia, Brasil.

10 Pontifícia Universidade Católica de Minas Gerais,

Belo Horizonte, Brasil.

11 Instituto de Estudos em Saúde Coletiva, Universidade Federal do Rio de Janeiro, Rio de Janeiro, Brasil.

12 Secretaria de Assistência à Saúde, Ministério da Saúde,

Brasília, Brasil.

13 Departamento de Medicina Social, Universidade Federal de

Pernambuco, Recife, Brasil.

14 Departamento de Odontologia Clínica e Preventiva

Universidade Federal de Pernambuco, Recife, Brasil.

15 Secretaria de Estado de Saúde de Minas Gerais, Belo

Horizonte, Brasil.

16 Faculdade de Odontologia, Universidade Paulista, São Paulo,

Brasil.

17 Universidade Metodista de São Paulo, São Paulo, Brasil.

18 Pontifícia Universidade Católica do Paraná, Curitiba, Brasil.

19 Universidade Federal do Paraná, Curitiba, Brasil.

20 Escola de Saúde Pública, Secretaria Estadual de Saúde,

Goiânia, Brasil.

21 Secretaria Municipal de Saúde de Goiânia, Goiânia, Brasil.

\section{Agradecimentos}

Os autores agradecem às 131 contribuições dadas ao projeto na consulta pública, às instituições que apoiaram o projeto e às secretarias estaduais e municipais das cidades participantes da amostra. Ao Fundo Nacional de Saúde.

\section{Referências}

1. Narvai PC. Saúde bucal coletiva: caminhos da odontologia sanitária à bucalidade. Rev Saúde Pública 2006; 40(N Esp):141-7.

2. Divisão Nacional de Saúde Bucal, Ministério da Saúde. Levantamento epidemiológico em saúde bucal: Brasil, zona urbana. Brasília: Ministério da Saúde; 1986.

3. Roncalli AG. Levantamentos epidemiológicos em saúde bucal no Brasil. In: Antunes JLF, Peres MA, organizadores. Epidemiologia da saúde bucal. Rio de Janeiro: Guanabara-Koogan; 2006. p. 32-48.

4. Narvai PC. O Brasil e as metas OMS-2000. Rev ABO Nac 2000; 7:374-7.

5. World Health Organization. Oral health surveys: basic methods. $4^{\text {th }}$ Ed. Geneva: World Health Organization; 1997.

6. Narvai PC, Antunes JLF, Moysés SJ, Frazão P, Peres MA, Peres KG, et al. Validade científica de conhecimento epidemiológico gerado com base no estudo Saúde Bucal Brasil 2003. Cad Saúde Pública 2010; 26:647-70.
7. Statistic Division, Department of Economic and Social Affairs, United Nations. Household sample surveys in developing and transition countries. New York: United Nations Publications; 2005.

8. Instituto Brasileiro de Geografia e Estatística. Manual do recenseador - CD 1.09. Rio de Janeiro: Instituto Brasileiro de Geografia e Estatística; 2000.

9. Kish L. Survey sampling. New York: John Wiley \& Sons; 1965.

10. Cochran WG. Sampling techniques. $3^{\text {rd }}$ Ed. New York: John Wiley and Sons; 1977.

11. Silva NN. Amostragem probabilística. 2a Ed. São Paulo: Edusp; 2001.

12. Departamento de Atenção Básica, Secretaria de Atenção à Saúde, Ministério da Saúde. Projeto SB Brasil 2003: condições de saúde bucal da população brasileira 2002-2003: resultados principais. Brasília: Ministério da Saúde; 2004. (Série C. Projetos, Programas e Relatórios). 
13. Macro International Inc. Sampling manual, DHSIII basic documentation No. 6. Calverton: Macro International Inc. 1996.

14. Holmgren C. CPITN: interpretations and limitations. Int Dent J 1994; 44:533-46.

15. World Health Organization. Oral health surveys: basic methods. 3rd Ed. Geneva: World Health Organization; 1987.

16. Cons NC, Jenny J, Kohout FJ, Songpaisan Y, Jotikastira D. Utility of the dental aesthetic index in industrialized and developing countries. J Public Health Dent 1989; 49:163-6.

17. Foster TD, Hamilton MC. Occlusion in the primary dentition: study of children at $2 \frac{1 / 2}{2}$ to 3 years of age. Br Dent J 1969; 21:76-9.

18. Fejerskov O, Manji F, Baelum V, Möeler IJ. Fluorose dentária: um manual para profissionais de saúde. São Paulo: Santos; 1994.
19. Dean HT. Classification of mottled enamel diagnosis. JAMA 1934; 21:1421-6.

20. Gil C, Nakamae AEM. Índice de qualidade do trabalho protético (IQP): um estudo metodológico. Revista da Pós-graduação 2000; 7:38-46.

21. Frias AC, Antunes JLF, Narvai PC. Precisão e validade de levantamentos epidemiológicos em saúde bucal: cárie dentária na Cidade de São Paulo, 2002. Rev Bras Epidemiol 2004; 7:144-54.

22. World Health Organization. Calibration of examiners for oral health epidemiological surveys. Geneva: World Health Organization; 1993.

Recebido em 11/Jan/2011

Versão final reapresentada em 13/Abr/2011

Aprovado em 04/Jul/2011 